\title{
ESPAก̃A Y ESTADOS UNIDOS: Un ANÁLISIS CONTEMPORÁnEO DE UNA RELACIÓn CONTROVERTIDA
}

\author{
Spain and the United States: A Contemporary \\ Analysis of a Controversial Relationship
}

\section{Juan Tovar Ruiz \\ Universidad de Burgos \\ E-mail: jtovar@ubu.es \\ 0 Autor}

La relación España-EE. UU. ha sido una de las más importantes desde la perspectiva de la política exterior española. A pesar de su carácter controvertido por la generación de diferentes disensos en las fuerzas políticas y de la relativa falta de reconocimiento oficial por parte de los líderes españoles, su naturaleza vital es clara debido a los importantes intereses en juego para España. El artículo pretende analizar la relevancia estratégica de la relación, las rupturas y continuidades producidas en las dos últimas décadas y las consecuencias de los últimos cambios políticos para el futuro de la relación bilateral.

The interstate relation Spain-United States has been on of the most relevant from the Spanish Foreign Policy perspective. Despite its controversial nature due to the dissensus it has leaded to among the Spanish political parties and the lack of recognition of its relevance from the Spanish strategic documents, its vital nature is clear given the relevant interests at stake for Spain. The article aims to analyze the strategic relevance of this relation, the ruptures and continuities of the last two decades and the consequences of the last political changes in Spain and the United States for the future of the bilateral relationship.

Política exterior; España; Estados Unidos; seguridad internacional; Administración Trump. Foreign policy; Spain; United States; international security; Trump Administration.

回 Key uords
Resumen

Abstract Fechas 


\section{Introducción y marco analítico}

El 16 de julio de 2018, los reyes de España visitaron oficialmente la Casa Blanca, reuniéndose con el presidente estadounidense Donald Trump y con la primera dama. Unos días más tarde, el presidente del Gobierno español, Pedro Sánchez, recibió una carta remitida por el presidente estadounidense, al igual que sucedió con otros Estados europeos que no cumplen con el objetivo fijado en la cumbre de Cardiff de 2014, de gastar el 2\% del PIB en defensa. En la citada carta, además de tomar en consideración la profunda crisis económica que atravesó España desde 2008 y de congratularse por la recuperación, mostró el creciente interés por el flanco sur demostrado, en teoría, por la OTAN y solicitó cumplir los compromisos previos de gasto en defensa, a los que su predecesor, Mariano Rajoy, se había comprometido.

En la respuesta de Sánchez se reflejó la tradicional postura española de que la contribución española no puede fundamentarse solo en una cifra de gasto, sino también en la "disponibilidad de capacidades y la voluntad de utilizarlas en favor de la paz y la seguridad internacionales". Después de los reproches del presidente Trump a sus aliados en el marco de la cumbre de la OTAN de julio de 2018, esta postura fue modificada y se volvió al planteamiento oficial de su predecesor, Mariano Rajoy, sobre el incremento del gasto en defensa (González y Abellán, 2018).

Este intercambio reciente es el último relativo a unas relaciones tradicionalmente controvertidas, pero de vital importancia para España y su política exterior. ¿Qué lecciones podemos extraer sobre ellas y qué nos dicen de la relación bilateral entre ambos Estados?

Para analizar las relaciones bilaterales España-EE. UU. el artículo se inspira en diferentes corrientes teóricas. En primer lugar, cabe destacar la corriente teórica del realismo neoclásico, esta es una variante del realismo político que ha tenido una particular utilidad en tiempos recientes a efectos de analizar la política exterior de los actores estatales del sistema internacional. En este aspecto, es una corriente que aúna en su planteamiento teórico la variable independiente del sistema internacional, en la que se centraban corrientes realistas previas como el realismo clásico o el neorrealismo, con los diferentes aspectos de la política interna a la hora de analizar la política exterior del Estado. Cabe destacar que, si bien originalmente el realismo neoclásico se había fundamentado en aspectos como la movilización de recursos, ha experimentado una evolución en este sentido incorporando otros aspectos como las tradiciones estratégicas de la política exterior, las preferencias de las élites, la opinión pública o la importancia del proceso decisorio (Lobell, Ripsman, y Taliaferro, 2016, pp. 161-188).

Otro de los aspectos destacables en un estudio de estas características que se debería tener en cuenta a la hora de profundizar en el análisis sobre esta materia es el del análisis de política exterior. Esta es una reciente subdisciplina de las relaciones internacionales y de la ciencia política que se ha centrado tradicionalmente en el proceso de toma de decisiones y que ha generado diferentes modelos ejemplificados por el clásico de Allison (1988) fundamentados en la existencia de un actor racional unificado, la importancia de la organización en el proceso de decisorio o de la competencia de actores y agencias que componen la Administración en concreto.

A pesar de la existencia de críticas de los planteamientos de unos y otros a sus respectivos posicionamientos teóricos, a juicio del autor no existe una incompatibilidad de base que impida utilizar elementos provenientes de ambas, especialmente si se toma la primera vertiente con cierta flexibilidad a la hora de respaldar el análisis. En cualquier caso, cabe plantear que la 
naturaleza de este trabajo no se circunscribe necesariamente de manera plena y exclusiva en ninguna variante teórica en concreto.

Un segundo aspecto de importancia en el análisis es el que se refiere a los diferentes órganos decisorios. En este aspecto, existe una cierta asimetría en el análisis del proceso decisorio y de los órganos que forman parte de él por comparación entre el caso estadounidense y el español. Mientras que en el supuesto estadounidense los diferentes órganos han sido estudiados de manera bastante minuciosa, el estudio en España de sus propios órganos decisorios ha sido muy inferior. Esto es un aspecto relativamente lógico dada la diferencia de posición entre Estados Unidos como primera potencia del sistema internacional y el Reino de España, una potencia media con ambiciones y recursos más modestos a la hora de desarrollar una política internacional.

En el caso de Estados Unidos, entre los órganos protagónicos de su política exterior destacan la figura del presidente como decisor último, comandante en jefe y jefe de la diplomacia, con mayores competencias brindadas por la Constitución que en otros ámbitos. Este tiene alrededor un órgano de creciente poder que es el Consejo de Seguridad Nacional, encargado del asesoramiento y coordinación de la Administración en materia de política exterior y de seguridad. Un órgano tradicionalmente relevante ha sido el Departamento de Estado, si bien se ha criticado la reducción de su influencia en tiempos recientes frente a otros organismos como el Departamento de Defensa o el propio Consejo de Seguridad Nacional. En cualquier caso, es la entidad que se ocuparía de la representación exterior del Estado y que tiene en su seno la USAID, entidad encargada de las políticas de cooperación internacional para el desarrollo en Estados Unidos. Junto a estos cabe destacar el propio Departamento de Defensa, de creciente importancia para su política exterior y las agencias de inteligencia. Fuera de los organismos ejecutivos quedaría el Congreso estadounidense, con competencias en política exterior que irían desde las labores de control hasta el establecimiento de sanciones, el control del presupuesto o la firma de tratados internacionales. Igualmente cabe destacar la opinión pública, los lobbies o grupos de interés privado y los think tanks como generadores de ideas (Brown y Snow, 2000, pp. 95-271; Rothkopf, 2004, pp. 7-10).

En el caso español cabe destacar que la relativa escasez de estudios sobre el proceso decisorio en política exterior ha llevado a una cierta centralización del rol del Ministerio de Asuntos Exteriores, que parece haber seguido teniendo - a diferencia del Departamento de Estado - un rol fundamental en el desarrollo de la política exterior más allá de la ejecución de la misma y a pesar de fenómenos como la globalización y el desarrollo tecnológico, que han tenido una importante incidencia en la realización de sus funciones. Menos estudios existen, a pesar de la relevancia que le otorga la Constitución Española, sobre la presidencia del Gobierno y sus funciones en la materia, planteándose a priori que su relevancia podría venir determinada antes por el interés del presidente del Gobierno en los asuntos internacionales que en las funciones constitucionalmente encomendadas. La figura del rey y su rol en la política exterior ha sido estudiada de manera más pormenorizada en tiempos recientes y, en lo que respecta a la relación bilateral con Estados Unidos, ha podido ejercer un rol de manera destacada en la materia. Asimismo, el Ministerio de Defensa también tiene un lugar destacado en la materialización de la política exterior y en las relaciones con la potencia norteamericana, dado el interés existente en cuestiones de seguridad o en activos como las bases de Morón y Rota, con presencia estadounidense. La Ley 16/2014 de 4 de diciembre de Acción Exterior (España, 2014), también resalta el papel protagónico de otras entidades como serían el Congreso de los Diputados, que ve reforzada su labor de control, las Comunidades Autónomas e incluso la Unión Europea, que

\section{La figura del rey y su rol en la política exterior ha sido estudiada de manera más pomenorizada en tiempos recientes}


dispone de su propio servicio de acción exterior. También los elementos derivados de la propia sociedad civil podrán tener un rol de importancia en la configuración de la política exterior española (Molina y Rodrigo, 2002, pp. 173-220; Priego, 2014, pp. 60-64; Tovar, 2018, pp. 84-90).

Si existe asimetría en el ámbito de los órganos de toma de decisiones, esta es mucho más notoria en el ámbito de las visiones del mundo. En tanto que en Estados Unidos grupos ideológicos como los realistas, los liberales intervencionistas, los neoconservadores o, en la actualidad, los jacksonianos, han tenido un papel importante a la hora de fijar las prioridades de la agenda estadounidense de política exterior, en España no ha existido un fenómeno similar, pese a los "escarceos" de algunos asesores del presidente del Gobierno José María Aznar con el neoconservadurismo y las divisiones se podrían haber realizado de manera más sencilla entre aquellos interesados en cuestiones internacionales y aquellos que no. El único "gran" debate que podríamos encontrar al respecto sería el referido al consenso y el disenso, esto es, el del acuerdo o desacuerdo de las principales fuerzas políticas en relación a la política exterior como política de Estado. Tampoco existen corrientes de pensamiento como las identificadas por Walter Mead en Estados Unidos: wilsonianos, jeffersonianos, jacksonianos y hamiltonianos. En este aspecto cabe destacar que no solo las visiones del mundo son relevantes, sino la posición de quien la sostiene en el ámbito del proceso decisorio (Walter Mead, 2002, pp. 86-96; Iglesias Cavicchioli, 2017; Arenal, 2008, pp. 3-6; Packer, 2005, p. 41).

Las regiones estratégicas prioritarias para ambos Estados y la diferencia en cuanto al nivel de poder existente son dos elementos añadidos a tener en cuenta a la hora de analizar la relación bilateral. En el caso estadounidense, tal y como se recogen en sus documentos estratégicos destacarían las de Asia-Pacífico, Europa y el Próximo Oriente. En los dos primeros casos, la presencia de grandes potencias con ciertas aspiraciones como son China o Rusia y, en el tercero, la relevancia — decreciente para la potencia norteamericana - energética y los desafíos de seguridad planteados por el yihadismo constituyen la base de este interés. En cualquier caso, por su condición de primera potencia del sistema internacional, Estados Unidos tiene capacidad y recursos para intervenir en cualquier región del planeta. En el caso de España, sus mucho más limitadas capacidades y recursos hacen que se centre en las regiones más próximas o a las que pertenece como Europa o el Mediterráneo o en aquellas con las que posee vínculos históricos o culturales como Latinoamérica, definida como Iberoamérica en la terminología diplomática española. Este es un aspecto que claramente diferencia y caracteriza a Estados Unidos como gran potencia frente al caso de España, considerada como un prototipo de potencia media (Casa Blanca, 2017, pp. 45-51; Tovar, 2018, pp. 90-96).

Este artículo no pretende ser un mero análisis histórico ni aportar un hecho desconocido, máxime cuando esta es, probablemente, la dimensión más estudiada de la relación bilateral, especialmente en etapas como la de la transición. Muy al contrario, su objetivo es el de tratar de responder tres preguntas concretas acerca de la relación bilateral entre España y Estados Unidos centrándose en la etapa actual:

1. ¿Cuál es la posición de Estados Unidos entre las prioridades estratégicas españolas de política exterior y a la inversa?

2. ¿Cuáles son las líneas de ruptura y continuidad existentes en la relación bilateral durante la última década?

3. ¿Qué efectos tiene para España y la relación bilateral la llegada del presidente Trump al poder y el desarrollo de sus planteamientos en materias como la defensa o el comercio? 
Para ello el artículo se divide en diferentes partes. La primera hace referencia a los fundamentos utilizados para realizar el análisis. La segunda hace un breve recorrido histórico por la relación bilateral centrándose en los momentos más recientes. La tercera analizará los principales documentos estratégicos de ambos países para estudiar la relevancia de la relación bilateral. Finalmente se expondrán las conclusiones y se tratará de dar respuesta a las preguntas planteadas.

\section{Evolución histórica de la relación bilateral}

Vistos los aspectos introductorios y básicos, conviene analizar de manera sucinta el recorrido histórico de la relación bilateral.

La relación entre España y Estados Unidos se remonta al propio origen de la potencia norteamericana, donde España participó de manera activa pese a las reticencias iniciales en el proceso de independencia que culminaría en 1783 con el Tratado de Versalles. Este es un aspecto hasta hace poco tiempo no demasiado conocido, pero que ha sido recuperado recientemente con la concesión de honores a alguno de los símbolos históricos en España de este periodo como es el general Bernardo de Gálvez (Obama y Felipe VI, 2015). Más tarde la anexión parcialmente forzada de Florida y la expansión hacia el sudoeste harían que Estados Unidos abarcase grandes territorios que antaño pertenecieron a la Corona española (García Cantalapiedra, 2010, pp. 441-443).

Las grandes desavenencias producidas a raíz del caso de Cuba y la posterior guerra de 1898, darían inicio a una cierta etapa de antiamericanismo que se mantendría con carácter intermitente y latente durante etapas históricas posteriores. Esta relación tirante volvería a ponerse de manifiesto tras la Segunda Guerra Mundial, con el aislamiento del régimen franquista. Sin embargo, las necesidades de la Guerra Fría y la consiguiente política de contención puesta en marcha por Truman, a inspiración parcial de la obra de Kennan y más tarde continuada por sus sucesores, hizo que el presidente Eisenhower iniciase un acercamiento que llevó al establecimiento de bases militares estadounidenses en España y al inicio de la asistencia económica, tecnológica y militar al régimen franquista en un marco de desequilibrio. La progresiva mejora para las necesidades en todos los aspectos anteriores de España, no llegarían sino de manera muy posterior y no se completarían hasta la entrada en democracia y los años 80 . Hay quien además vincula el resurgimiento de un cierto antiamericanismo, esta vez en la izquierda política, debido al apoyo de Estados Unidos al régimen franquista durante este periodo histórico (García Cantalapiedra, 2010, pp. 443-449; Crespo Palomares, 2016, pp. 21-36; Marquina, 1986).

A pesar de importantes tradiciones políticas desde la perspectiva de la política exterior estadounidense como el idealismo wilsoniano y su idea de "hacer el mundo seguro para la democracia”, puesta en marcha tanto en el periodo de entreguerras como en la posguerra fría, algunos investigadores no han observado en la cautelosa posición estadounidense hacia la transición en España, un apoyo decidido en favor de la democracia. La prioridad a este respecto, de importantes administraciones como las de los presidentes Nixon y Ford, fue siempre conservar el importante activo de las bases militares, máxime en el contexto de la competición bipolar resultado de la Guerra Fría. Otros autores, en cambio, sostienen que el fortalecimiento y la consolidación democrática de España sí que habría podido tener un cierto papel en el marco de las acciones estadounidenses durante este periodo. En cualquier caso, los pronunciamientos de algunos dirigentes estadounidenses en momentos clave de la transición como los del secretario de Estado, Alexander Haig, de cara al 23-F, no contribuyeron a un mayor entendimiento

\section{La progresiva mejora para las necesidades de España no se completarían hasta la entrada en democracia y los años 80,junto con un resurgimiento de un cierto antiamericanismo}


con las fuerzas más críticas con la potencia norteamericana (Powell, 2014, pp. 28-34; Crespo Palomares, 2016, pp. 32-34).

La relación con Estados Unidos marcaría los grandes disensos de la política exterior española durante la democracia, si bien no todos los autores están de acuerdo con la existencia de la figura del consenso en esta política pública. Concretamente, dos de estos momentos serían el de la entrada de España en la OTAN y el del debate sobre la guerra de Irak. La decisión de entrar en la OTAN fue tomada por parte del Gobierno de Leopoldo Calvo-Sotelo en 1982, pero sería inicialmente rechazada por el Partido Socialista, que llegó a incluir en su programa electoral la convocatoria de un referéndum sobre la pertenencia de dicha organización. La creciente vinculación entre la entrada de España en la OTAN y en las Comunidades Europeas llevaría a un nuevo cambio de posición del Gobierno socialista, que acabaría apoyando la entrada en dicha organización con condiciones y contribuiría a la victoria de los partidarios de la membresía por un escaso margen (Rodrigo Rodríguez, 1995, pp. 77-103).

El segundo de los grandes momentos de disenso se produciría como consecuencia del apoyo de Aznar a la guerra de Irak de 2003, que generó una nueva división política y el nuevo crecimiento del antiamericanismo durante este periodo histórico. La posición de algunos críticos hacia dicha decisión se fundamentó en la ruptura del posicionamiento de Aznar con la tradicional visión española de preeminencia de regiones estratégicas como el Mediterráneo o Latinoamérica y, especialmente, con la prioridad fundamental de Europa. Esta crítica es solo parcialmente cierta, pues la realidad política europea del momento era de división y no tanto de alineamiento a favor o en contra de la posición estadounidense, por lo que es difícil sostener que ese alejamiento de una supuesta prioridad previa por Europa se llegó a producir realmente. Entre las motivaciones que llevaron al presidente del Gobierno a apoyar a la Administración estadounidense podrían encontrarse la debilidad de la posición española en Europa tras la ampliación al Este, el deseo de ejercer una mayor influencia y protagonismo en el sistema internacional de la mano de la primera potencia mundial o la defensa de los intereses nacionales españoles entendidos en el marco de la guerra contra el terror, un concepto que empatizaba con los tradicionales esfuerzos españoles en la lucha contra la banda terrorista ETA (Arenal, 2008, pp. 19-22; Crespo Palomares, 2016, pp. 251-253).

En cualquier caso, la victoria electoral en las elecciones de 2004 del Partido Socialista no llevaría a una mejora de las relaciones bilaterales entre España y Estados Unidos. A la crítica contundente a la guerra de Irak o a una retirada de los efectivos españoles poco coordinada se sumarían algunos errores diplomáticos ante símbolos estadounidenses y declaraciones que animaban a la retirada de las tropas aliadas de Irak que no agradarían en la Casa Blanca y que obligaron a la realización de notables esfuerzos posteriores para recuperar la fluidez de la relación bilateral, especialmente en lo que respecta a la relación personal entre el presidente estadounidense y el del Gobierno español. Aspectos como el incremento de tropas españolas en Afganistán y las dificultades para lograr un asiento para España en la primera de las grandes cumbres del G-20, celebrada en 2008 en Washington, así lo demuestran (Powell, 2009, pp. 523-526).

Paradójicamente, y a pesar de las críticas vertidas hacia la potencia norteamericana, el Gobierno de José Luis Rodríguez Zapatero contribuiría a una nueva mejora en la relación bilateral con las importantes decisiones tomadas durante su segunda legislatura.

Los intereses principales defendidos por Estados Unidos en España durante este periodo histórico se centrarían en 1 - las cuestiones económicas y comerciales, 2 - la política de seguridad y 
defensa, y 3 - la defensa de la propiedad intelectual. Los dos primeros tendrían una continuidad en etapas posteriores, la tercera perdería entidad con las reformas legislativas introducidas, principalmente, por el Gobierno de Rajoy para combatir las descargas ilegales (Tovar, 2016, p. 5; Koch y Marcos, 2013).

Tras la victoria del presidente Obama en las elecciones de 2008, el Gobierno español inició un acercamiento al nuevo presidente estadounidense, lo que permitiría después de una espera de años la materialización de una visita al despacho oval y una invitación al conocido como desayuno de la oración. Con todo, la decisión más importante, que marcaría una nueva continuidad en el ámbito de las relaciones bilaterales, sería el de la modificación del convenio de defensa con Estados Unidos de 2011, que permitiría el establecimiento de cuatro destructores estadounidenses que formaban parte del conocido popularmente como escudo antimisiles. De igual forma, España participaría, aunque de forma limitada, en la intervención en Libia de 2011 que provocó la caída del régimen de Gadafi (González y Martínez de Rituerto, 2011).

Estas decisiones llevarían a que, paradójicamente, un presidente del Gobierno que se había caracterizado en su origen por las importantes críticas a Estados Unidos y la dificultad para mantener la relación al más alto nivel político, acabaría convirtiéndose en el principal impulsor de la relación bilateral.

La política hacia Estados Unidos mantendría una clara continuidad con el presidente del Gobierno Mariano Rajoy. Si bien el centro de la atención española durante estos momentos de crisis estuvo en la Unión Europea, se mantuvieron relaciones cordiales con los dos presidentes estadounidenses con los que se coincidió: Barack Obama y Donald Trump. En el marco de la relación bilateral el hito más relevante fue el de la nueva modificación del convenio de defensa de 2015, que permitió el establecimiento de una fuerza de reacción rápida frente a una crisis en el continente africano y que estaría destacada en la base de Morón e incrementó la presencia estadounidense hasta un máximo posible de 2200 soldados, 500 empleados civiles y 36 aeronaves. Esta última modificación, que tiene su origen en la conocida crisis de Bengasi de 2011, resultaría en una nueva reivindicación más de la importancia que iban adquiriendo las bases estadounidenses en España. Una relevancia en el plano militar, que no parecía ser respaldada desde una perspectiva política con la mayor visibilidad de la relación bilateral (El Mundo, 2015).

Asimismo, se participaría de forma limitada en la coalición que luchaba para acabar con el Estado Islámico, se adquirió el compromiso de avanzar hacia el $2 \%$ en materia de defensa durante la cumbre de la OTAN en Cardiff en 2014 y se apoyó la importante iniciativa estadounidense de establecer un tratado transatlántico para el libre comercio y las inversiones, el conocido como TTIP, cuyas negociaciones despertaron más críticas en el ámbito europeo que en el estadounidense y que serían congeladas con la llegada del presidente Trump al poder. En el plano económico, Estados Unidos era en 2016 el principal inversor en España —un 16\% del total — y el principal destino de las inversiones españolas — $17 \%$ del total — y el principal socio comercial fuera de la Unión Europea (Icex, 2017). También se avanzaría en el reconocimiento cultural de símbolos de la relación bilateral, con la visita de los reyes de septiembre de 2015 , cuando se recibirían del presidente Obama los honores del militar y político español, Bernardo de Gálvez (Obama y Felipe VI, 2015).

Una de las razones que permitirían explicar esta falta de interés en la acción de visibilizar de la relación bilateral por parte estadounidense sería la de la prioridad otorgada por la Administra- 
ción estadounidense a la estrategia de "giro hacia el pacífico" y la relación con las emergentes frente a los aliados tradicionales europeos. Un aspecto que pudo ejemplificarse con la cancelación de la cumbre UE-EE. UU. prevista durante la presidencia española, que generó una cierta frustración en el Gobierno de Rodríguez Zapatero. Una prioridad que no comenzaría a modificarse hasta el estallido de las Primaveras Árabes. La otra razón que explicaría la falta de interés estaría en que dicha relación fuese percibida por parte de las autoridades estadounidenses como algo dado de antemano, siendo el caso español un supuesto claro que no implicaba ni problemáticas ni soluciones (Tovar, 2017, pp. 1-2).

Lo cierto es que, en tanto que el presidente estadounidense visitó de manera reiterada diferentes países europeos, España únicamente sería visitada por Obama en 2016, justo antes de finalizar su segundo mandato y tuvo una cierta sensación de despedida más que de oportunidad para afrontar los nuevos desafíos estratégicos que ambos Estados afrontaban. A pesar de todo, las declaraciones tanto del presidente estadounidense como de los destacados miembros de la Administración que visitaron España en este periodo fueron cordiales, llegando el secretario de Estado Kerry a calificar a España como "aliado estratégico" e incluso "vital" (La Vanguardia, 2015). El propio Obama declaró que "no podríamos pedir un aliado mejor que España” (Obama, 2016). Curiosamente, esta relación mantuvo su fluidez a pesar de la importante modificación del panorama político del país, en torno a cuatro fuerzas políticas, no todas necesariamente favorables a las iniciativas estadounidenses (Manetto, 2016).

La llegada al poder del presidente Trump, aparentemente, no parecía facilitar el diálogo bilateral ni despertaba, al igual que sucedía en el caso de otros aliados europeos, demasiadas simpatías. Su discurso durante la campaña de primarias, que se focalizó en las críticas contra los acuerdos de libre comercio o el déficit comercial que mantenía con Estados aliados y rivales o la consideración de la OTAN como una "organización obsoleta" no facilitaba a priori el entendimiento. De igual forma, su relativamente imprevista elección cogió desprevenido al Gobierno, cuyos representantes daban por hecha la victoria de la candidata demócrata Hillary Clinton, a cuya convención acudieron miembros destacados del mismo y tuvieron que encontrar nuevas formas de acercamiento al nuevo presidente estadounidense. El presidente Trump, además, haría del incremento del gasto en defensa, uno de los puntos principales de su política transatlántica, como se ejemplificó en la cumbre de la OTAN de mayo de 2017, que contó con la primera visita del presidente estadounidense. En este aspecto, España no estaba bien posicionada, pues su gasto estimado de $0,9 \%$ del PIB en defensa quedaba lejos de los objetivos fijados en la cumbre de Cardiff de 2014 y en uno de los últimos lugares de manera comparativa (Trump, 2017; Tovar, 2017, pp. 2-3).

A pesar de todo, estas relaciones fueron mejor de lo esperado y dentro del primer año de presidencia de Trump, en septiembre de 2017, se cursó una invitación para Mariano Rajoy a visitar el Despacho Oval. En el primer encuentro se trataron diferentes cuestiones de índole económica, comercial o de defensa. Puede observarse de manera destacada que el difícil contencioso de Cataluña tuvo también su papel aquí, respaldando el presidente Trump inequívocamente el posicionamiento español, yendo más allá respecto de algunos de los aliados europeos de España. Asimismo, tanto en la visita de Rajoy como en la de los reyes de junio de 2018, calificó la relación bilateral como "extraordinaria" en los diferentes niveles económico y comercial, cultural o de seguridad, cuestión también facilitada por un intercambio comercial bilateral favorable a Estados Unidos (Trump y Rajoy, 2017; Tovar, 2017, pp. 7-8; Trump y Felipe VI, 2018). 
En el plano del gasto en defensa, España se comprometió a incrementarlo, si no hasta el 2\% fijado en Cardiff, al 1,53\% para 2024. Esta posición fue confirmada por el Gobierno de Sánchez después de la controvertida cumbre de la OTAN de julio de 2018 (El País, 2018).

\section{La relación bilateral en los documentos estratégicos de España y Estados Unidos}

Una vez analizada la evolución de las relaciones bilaterales conviene ver, a efectos de sacar conclusiones, cuál es la presencia de ambas potencias en los documentos estratégicos respectivos. A estos efectos, podemos asumir de partida la existencia de una enorme asimetría entre la relevancia estratégica que tendría Estados Unidos para España y, en cambio, la menor dimensión de España para Estados Unidos dada su condición de primera potencia del sistema internacional.

En este sentido, los documentos estratégicos ofrecen una visión relativamente clara. Los documentos estratégicos que se exponen son solo los más recientes, correspondiendo en el caso de Estados Unidos con las Administraciones de Obama y Trump y en el español, con la etapa más reciente de los Gobiernos de José Luis Rodríguez Zapatero y los de Mariano Rajoy, dado que el Gobierno de Pedro Sánchez ha tenido un desempeño relativamente reciente y aún no hizo público un documento de tales características. En el caso estadounidense se han analizado las estrategias de seguridad nacional, los documentos más relevantes desde la perspectiva de la política exterior y de la seguridad nacional para la potencia norteamericana.

Las estrategias de seguridad nacional de los años 2010 y 2015, correspondientes a la presidencia de Obama, no tratan a España de manera individualizada. Su especificidad, al igual que la de otros Estados europeos quedaría subsumida en el marco europeo y, especialmente, en relación a dos organizaciones de especial interés para Estados Unidos: la Unión Europea y la OTAN. En el primer caso, en relación a políticas económicas y comerciales o de lucha contra el cambio climático y, en el segundo, de seguridad internacional. En la estrategia de 2010 menciona la actuación conjunta con los aliados europeos en escenarios como los Balcanes, Chipre o la cooperación con Turquía. En la de 2015 cabe tener en cuenta la especificidad de la incorporación de las negociaciones en torno al TTIP y del desafío ruso después de la anexión de Crimea y la crisis de Ucrania. En cualquier caso, la consideración de España sería la de aliado estadounidense pero subsumido dentro del marco europeo más amplio, situada en la estrategia de 2015 en segundo lugar después del caso de Asia, donde se resalta la estrategia de Rebalance, pese a que en 2010 aparecía en el primer lugar, destacada como "piedra angular" de la relación estadounidense con el mundo (Casa Blanca, 2010, pp. 41-42; Casa Blanca, 2015, p. 25).

En el caso de la estrategia de seguridad nacional de 2017, correspondiente a la Administración Trump, no hay una presencia española mucho más destacada. En este caso, la única mención realizada se hace, junto al caso de otros Estados europeos, en referencia a la vulnerabilidad existente frente a los ataques del terrorismo yihadista, uno de los desafíos comunes entre europeos y estadounidenses. Las cuestiones comerciales, establecidas en relación a acuerdos de libre comercio, justos y recíprocos, será otro de los aspectos más destacados dentro de este documento. Finalmente, y especialmente recalcada, está la cuestión del gasto del $2 \%$ en defensa, establecido en la cumbre de Cardiff de 2014. Este incremento parece una de las cuestiones centrales defendidas por la Administración estadounidense en relación a sus principales aliados y ha sido respaldada por los hechos en las diferentes cumbres producidas en 2017 y 2018 ,
Existen dos organizaciones de especial interés para Estados Unidos: la Unión Europea y la OTAn 
donde el presidente estadounidense ha reclamado a sus socios avances en esta cuestión. Este es un aspecto donde la posición española no es particularmente destacada, siendo uno de los países, como ya se ha comentado, en los que el gasto en la materia es relativamente menor (Casa Blanca, 2017, pp. 47-48).

Un cambio que puede observarse en esta estrategia respecto de las de su predecesor, es la mayor presencia de la dimensión bilateral de la relación si bien, mencionando y reiterando la necesidad de cooperar con la Unión Europea. El tratamiento es nuevamente el de Estados aliados y se mencionan algunos desafíos de seguridad del sistema internacional como las acciones de Rusia y los planes nucleares de Irán y Corea del Norte. La presencia europea se recoge en segundo lugar después de la región "indoasiática”.

En el caso español, se analizarán dos tipos de documentos. En primer lugar, las estrategias de seguridad nacional que los sucesivos Gobiernos han ido haciendo públicas desde el año 2011 y, en segundo lugar, la estrategia española de acción exterior que apareció en el año 2014. Queda al margen del análisis la consideración de la efectividad de dichos documentos y la naturaleza realmente estratégica.

En el caso de la estrategia de seguridad nacional de 2011, Estados Unidos aparece en segundo lugar entre las prioridades estratégicas después de Europa y por delante de Rusia. Este orden no parece precluir las prioridades estratégicas, teniendo en cuenta la mayor relevancia de regiones de interés tradicional para España como Latinoamérica o el Mediterráneo sobre la potencia euroasiática. De la relación transatlántica se destacan un amplio abanico de elementos que incluyen cuestiones científicas, económicas, culturales y de defensa con especial mención al convenio bilateral en la materia. También hay mención a desafíos como la seguridad de las conexiones marítimas y aéreas, la inmigración ilegal, el narcotráfico o la seguridad energética. Se hace mención a una dimensión transatlántica más amplia que incluya también a África y Latinoamérica (Moncloa, 2011, pp. 26-27).

La revisión realizada en 2013 de dicho documento, ya con el Gobierno de Mariano Rajoy, supone una clara continuidad en estos aspectos, si bien recogidos de una manera mucho más vaga y breve, y situados en el tercer lugar de prioridades, después de Europa, Latinoamérica y el Mediterráneo. También menciona brevemente la cuestión de la población hispana y continúa proponiendo la citada visión amplia de las relaciones transatlánticas (Moncloa, 2013, pp. 16-17).

Finalmente, en la versión de 2017 se menciona de manera muy escueta el papel jugado por la relación con Estados Unidos en materia de seguridad, especificando el convenio de defensa y la amenaza yihadista o los valores compartidos. Cabe destacar que la OTAN no es mencionada en este apartado sino como un instrumento de seguridad recogido en el ámbito europeo, no en el estadounidense que se subsume dentro del apartado denominado "América del Norte" y donde se hace referencia al desafío que supone la posición rusa en relación al conflicto de Ucrania. El lugar que ocupa la relación con Estados Unidos en esta versión está en el quinto lugar, detrás de las tres regiones mencionadas con anterioridad y África Subsahariana (Moncloa, 2017, pp. 43-44 y 51).

La estrategia española de acción exterior de 2014, el primer documento publicitado de este tipo y, paradójicamente, elaborado por el Ministerio de Asuntos Exteriores y no por la Moncloa, a pesar de su extensión y del exceso de detalle, que hace difícil establecer prioridades claras, también recoge alguna referencia a la relación con Estados Unidos. En este documento la relación bilateral es recogida con algo más de detalle, especificando sus diferentes dimensiones: política, económica, de seguridad y cultural, y mencionando algunos desarrollos coetáneos a la

\section{En el caso de} la estrategia de seguridad nacional de 2011 , Estados Unidos aparece en segundo lugar entre las prionidades estratégicas después de Europa y por delante de Rusia 
estrategia como el de las negociaciones de un acuerdo de libre comercio. También se menciona la cuestión de la comunidad hispana. El lugar que ocupa la posición estadounidense es, en este caso, el cuarto después de las tres regiones tradicionales (Ministerio de Asuntos Exteriores y Cooperación, 2014, pp. 124-126).

\section{Conclusiones}

A la vista de los desarrollos anteriores cabe plantear las conclusiones al trabajo en respuesta a las preguntas recogidas en la introducción del mismo. En primer lugar, en relación a la posición que ocupa cada uno de los dos actores dentro de las prioridades en política exterior del otro, cabe resaltar la enorme asimetría existente como consecuencia del diferente nivel de poder y relevancia en el marco del sistema internacional.

De tal forma, para comprender la relevancia de España en la política exterior estadounidense, esta ha de subsumirse dentro del entorno regional europeo. Esta ha sido la constante de las últimas presidencias estadounidenses y ha marcado una cierta continuidad entre la presidencia de Obama y la de Trump. Esto a pesar de las notables diferencias entre una primera Administración que prefería tratar a sus aliados europeos desde una perspectiva multilateral y una Administración Trump que prefiere la vía de las relaciones bilaterales. El ascenso de Asia y los importantes desafíos de seguridad en esa región parecen haber subsumido a Europa en un segundo lugar del orden de prioridades, si bien y a pesar de las actuales diferencias en numerosas políticas, es el lugar donde gran parte de sus aliados están presentes. En lo que respecta a España y situándonos dentro de este marco, su relevancia en materia de seguridad o defensa, a pesar de importantes hándicaps como el escaso gasto en defensa por PIB, ha incrementado su relevancia en materia de seguridad por la importancia de la presencia de bases militares clave para la proyección de poder como Morón y Rota, manteniéndose además los intereses económicos y comerciales que la potencia estadounidense ha desarrollado en España como parte de la UE, en tanto las cuestiones de propiedad intelectual han reducido su relevancia.

En el caso de España, la relación no puede ser considerada sino "vital". A pesar de que su relevancia ha sido postergada en el discurso de sus líderes y en sus principales documentos estratégicos, la dependencia de la potencia norteamericana en términos de seguridad, máxime en momentos de escaso gasto en defensa y en un contexto en el que el desarrollo de la defensa europea todavía no ha disipado las dudas que permitirían considerarla una posible alternativa, hace que no pueda ser considerada sino de otra manera. A esto hay que añadir los importantes intereses económicos, comerciales y culturales que España tiene en Estados Unidos como su principal inversor y cliente fuera de la Unión Europea. A la inversa, las cifras serían más modestas. Las razones del no reconocimiento por parte de los líderes españoles pueden darse en la pervivencia de cierto antiamericanismo en algunos sectores ideológicos, críticos con las posiciones estadounidenses.

La segunda pregunta trata las rupturas y continuidades presentes en la relación bilateral durante los últimos años. En este aspecto podemos constatar que las continuidades son más importantes que las rupturas. En el caso estadounidense, tanto las prioridades como el discurso han tenido escasos cambios, si bien puede destacarse la existencia de una reivindicación más enérgica de planteamientos como el incremento del gasto en defensa, presente en las demandas de la Administración Obama, pero defendidas de manera más vocal por la Administración Trump en foros bilaterales y multilaterales. En el caso español, incluso remontándonos a la 
segunda legislatura del presidente del Gobierno José Luis Rodríguez Zapatero y su "giro atlantista”, la relación bilateral ha quedado marcada por la continuidad sin la presencia de notorios disensos como los protagonizados en 1982-85 o 2003 pese a la aparición de nuevas fuerzas políticas, alguna con posicionamientos críticos hacia propuestas estadounidenses, como sucedió con Podemos y el TTIP. Ni siquiera ante la moción de censura del 1 de junio de 2018 y las circunstancias que llevaron al presidente Pedro Sánchez al poder, esta continuidad parece haberse roto y, de manera más clara, parece haberse seguido una postura de continuidad respecto de los Gobiernos de Mariano Rajoy, asumiendo una postura de neutralidad, que no llevó a España a alinearse con la Administración estadounidense, ni con los Estados europeos más críticos como Alemania.

Con todo, las crecientes desavenencias transatlánticas en diferentes políticas como las de comercio o defensa, en un marco de establecimiento mutuo de aranceles al comercio o de críticas por el insuficiente gasto en defensa hacen que no puede descartarse la aparición de un nuevo debate político sobre la relación bilateral. Eventos como los acontecidos en el marco de la cumbre del G-7 del 8 de junio de 2018 o de la cumbre de la OTAN del 12 y 12 de julio de 2018 son ejemplificativos de dicha posibilidad.

Desde la perspectiva del autor, está en el interés español poner en valor la importancia de una relación que tiene una clara naturaleza vital, por encima de la persona que ocupe coyunturalmente el cargo de presidente de los Estados Unidos. Esto no se debe producir en perjuicio de la defensa clara de los intereses españoles cuando estos no coincidan con los estadounidenses, como sucede con las cuestiones comerciales, y su defensa a través de mecanismos propios o bien de la Unión Europea, pero el retorno a un nuevo debate político innecesario y estéril sobre la misma, produciría más perjuicios que beneficios para los intereses de España como potencia media y debilitaría sus opciones a la hora de hacer valer su posición e intereses en las batallas por venir en diferentes instancias, incluyendo la de la defensa europea.

\section{Bibliografía}

Arenal, C. del (2008). Consenso y disenso en la política exterior de España. Real Instituto Elcano. Recuperado de http://www.realinstitutoelcano.org/wps/portal/rielcano_es/ contenido?WCM_GLOBAL_CONTEXT=/elcano/elcano_es/zonas_es/europa/dt25-2008

Brown, E., y Snow, D. M. (2000). United States Foreign Policy. Politics Beyond the Water's Edge. St. Martin's Boston: Bedford.

Casa Blanca (2010). National Security Strategy. Recuperado de http://nssarchive.us/NSSR/2010.pdf

Casa Blanca (2015). National Security Strategy. Recuperado de http://nssarchive.us/wp-content/uploads/2015/02/2015.pdf

Casa Blanca (2017). National Security Strategy. Recuperado de https://www.whitehouse.gov/ wp-content/uploads/2017/12/NSS-Final-12-18-2017-0905.pdf

Crespo Palomares, C. (2016). La alianza americana. La estrategia antiterrorista española y las relaciones hispano-norteamericanas (1996-2004). Madrid: Catarata.

El mundo (29 de mayo de 2015). Morón será base permanente de EE. UU. para operaciones en África. El Mundo. Recuperado de http://www.elmundo.es/andalucia/2015/05/29/556 878e5268e3e97518b458d.html 
España (2014). Ley Orgánica 2/14, de 25 de marzo, de la Acción y del Servicio Exterior del Estado. Boletín Oficial del Estado. Recuperado de https://www.boe.es/buscar/act. php?id=BOE-A-2014-3248

García Cantalapiedra, D. (2010). España, Estados Unidos y las relaciones transatlánticas. En J. C. Pereira (ed.), La política exterior de España. De 1800 hasta hoy (pp. 441-458). Barcelona: Ariel.

González, M., \& Martínez de Rituero, R. (5 de octubre de 2011). EE. UU. desplegará en Rota 1.100 militares y cuatro buques del escudo antimisiles. El País. Recuperado de https:// elpais.com/politica/2011/10/05/actualidad/1317802582_270373.html

González, M., \& Abellán, L. (12 de agosto de 2018). Sánchez asume el objetivo de aumentar el gasto militar al 2\% del PIB. El País. Recuperado de https://elpais.com/internacional/2018/07/12/actualidad/1531404525_333392.html

ICEX (2017). España. Exportación e inversiones. Estados Unidos. Recuperado de https://www. icex.es/icex/es/navegacion-principal/todos-nuestros-servicios/informacion-de-mercados/ paises/navegacion-principal/el-pais/relaciones-bilaterales/index.html?idPais=US

Iglesias Cavicchioli, M. (2017). Aznar y los "neocon". El impacto del neoconservadurismo en la politica exterior de España. Barcelona: Huygens.

Koch, T., \& Marcos, A. (1 de mayo de 2013). España se salva de volver a la lista negra de la piratería de EE. UU. El País. Recuperado de https://elpais.com/cultura/2013/04/24/actualidad/1366815605_846713.html

La Vanguardia (9 de octubre de 2015). Kerry felicita al pueblo español por la fiesta nacional. La Vanguardia. Recuperado de https://www.lavanguardia.com/politica/20151009/54438014903/kerry-felicita-al-pueblo-espanol-por-la-fiesta-nacional.html

Lobell S. E., Ripsman, N. M., y Taliaferro, J. W. (2016). Neoclassical Realist Theory of International Politics. Nueva York: Oxford University Press.

Manetto, F. (9 de mayo de 2016). Podemos hará de la oposición al TTIP un "tema frontal de campaña”. El País. Recuperado de https://elpais.com/politica/2016/05/09/actualidad/1462791504_069597.html

Marquina Barrios, A. (1986) España en la política de seguridad occidental. Madrid: Ejército.

Mead, W. R. (2000). Special Providence. American Foreign Policy and How it Changed the World. Nueva York: Routledge.

Ministerio de Asuntos Exteriores y Cooperación (2014). Estrategia Española de Acción Exterior. Recuperado de http://intercoonecta.aecid.es/Documentos\%20de\%20la\%20comunidad/ Estrategia_Acci\%C3\%B3n\%20Exterior-2014.pdf

Molina, I., \& Rodrigo, F. (2002). Las transformaciones organizativas de la política exterior española. Revista de Estudios Políticos, (117), 173-220.

Moncloa (2011). Estrategia de Seguridad Nacional. Recuperado de http://www.realinstitutoelcano.org/wps/wcm/connect/c06cac0047612e998806cb6dc6329423/EstrategiaEspanolaDeSeguridad.pdf?MOD=AJPERES\&CACHEID=c06cac0047612e998806cb6 dc6329423 
Moncloa (2013). Estrategia de Seguridad Nacional. Recuperado de http://www.lamoncloa.gob. es/documents/seguridad_1406connavegacionfinalaccesiblebpdf.pdf

Moncloa (2017). Estrategia de Seguridad Nacional. Recuperado de http://www.lamoncloa.gob. es/documents/seguridad_1406connavegacionfinalaccesiblebpdf.pdf

Obama, Barack y Felipe VI (2015). Remarks by President Obama and His Majesty King Felipe of Spain After Bilateral Meeting. Recuperado de https://obamawhitehouse.archives. gov/the-press-office/2015/09/15/remarks-president-obama-and-his-majesty-king-felipespain-after

Obama, Barack (2016). Remarks by President Obama to Troops at Naval Station Rota. Recuperado de https:/obamawhitehouse.archives.gov/the-press-office/2016/07/10/remarkspresident-obama-troops-naval-station-rota

Packer, G. (2005). The Assasins' Gate. America in Iraq. Nueva York: Farrar, Strauss \& Giroux.

Powell, C. (2014). El amigo americano. España y Estados Unidos de la dictadura a la democracia. Barcelona: Galaxia Gutenberg.

Powell, C. (2009). A Second Transition, or More of the Same? Spanish Foreign Policy under Zapatero. South European Society and Politics, 14(4), 519-536. DOI: https://doi. org/10.1080/13608740903503886

Priego, A. (2014). La Corona en la diplomacia (pública) española. Comillas Journal of International Relations, (1), 54-79. DOI: https://doi.org/10.14422/cir.i01.y2014.005

Rothkopf, D. (2004). Running the World. The Inside Story of the National Security Council and the Architects of American Power. Nueva York: Public Affairs.

Rodrigo Rodríguez, F. (1995). La inserción de España en la política de seguridad occidental. En R. Gillespie, J. Story, y F. Rodrigo, (eds.), Las relaciones exteriores de la España democrática (pp. 77-103). Madrid: Alianza Editorial.

Tovar Ruiz, J. (2016). Las relaciones España-EE. UU. en tiempos de incertidumbre. Real Instituto Elcano. Recuperado de http://www.realinstitutoelcano.org/wps/portal/web/rielcano_es/contenido?WCM_GLOBAL_CONTEXT=/elcano/elcano_es/zonas_es/ari542016-tovarruiz-relaciones-espana-eeuu-tiempos-incertidumbre

Tovar Ruiz, J. (2017). Las relaciones España-EE. UU. en los albores de la era Trump. Real Instituto Elcano. Recuperado de http://www.realinstitutoelcano.org/wps/portal/rielcano_es/contenido?WCM_GLOBAL_CONTEXT=/elcano/elcano_es/zonas_es/eeuudialogo+trasatlantico/ari74-2017-tovarruiz-relaciones-espana-eeuu-era-trump

Tovar Ruiz, J. (2018). Retos y desafíos de la política exterior y de seguridad española: toma de decisiones y regiones estratégicas. En Seguridad Integral Global. Análisis del Derecho en las situaciones de crisis humanitarias (pp. 81-106). Madrid: Ministerio de Defensa.

Trump, D. (2017). Remarks by President Trump at NATO Unveiling of the Article 5 and Berlin Wall Memorials-Brussels, Belgium. Recuperado de https://www.whitehouse.gov/briefings-statements/remarks-president-trump-nato-unveiling-article-5-berlin-wall-memorials-brussels-belgium/ 
Trump, D., y Felipe VI (2018). Remarks by President Trump and His Majesty King Felipe VI of Spain Before Bilateral Meeting. Recuperado de https:/www.whitehouse.gov/briefingsstatements/remarks-president-trump-majesty-king-felipe-vi-spain-bilateral-meeting/

Trump, D., y Rajoy, M. (2018). Remarks by President Trump and President Rajoy of the Government of Spain in Joint Press Conference. Recuperado de https://www.whitehouse.gov/ briefings-statements/remarks-president-trump-president-rajoy-government-spain-jointpress-conference/ 